\title{
GOBERNACIÓN DEL CAMBIO CLIMÁTICO EN MÉXICO: EXPECTATIVAS DE REFORMAS EN EL CONTEXTO SUB-NACIONAL
}

\author{
Ricardo V. Santes-Álvarez ${ }^{1}$
}

\begin{abstract}
RESUMEN
En este artículo se revisa la gobernación del cambio climático de México y las perspectivas de realizar reformas en espacios sub-nacionales. Siguiendo la orientación del ámbito externo, el gobierno federal ha asumido el reto de enfrentar la variación del clima con la convicción de ser capaz de, por un lado, liderar el mundo en desarrollo y, por otro, promover reformas a la administración pública que repercutan positivamente en las distintas demarcaciones territoriales. Las iniciativas gubernamentales han respondido a una estrategia abarcadora que gira en torno al sistema de acción frente al cambio climático; empero, la complejidad de la problemática rebasa planteamientos centralistas, convocando a la participación de gobiernos estatales y municipales, cuyas capacidades institucionales son heterogéneas, por lo que deben fortalecerse para generar desempeños locales y arreglos intergubernamentales eficientes. El análisis de documentos normativos e instrumentos de política pública, revela la pertinencia de instrumentar reformas a la gobernación del cambio climático en México. En esa perspectiva, la atención del fenómeno demanda una política con visión de integralidad, así como la consideración de dos tareas pendientes: descentralización y priorización de acciones en regiones vulnerables. En el interés de satisfacer los objetivos de la sustentabilidad social y ambiental, México debe construir un tipo de gobernación que promueva la coordinación intergubernamental e intersectorial, la participación amplia de los actores, y la vigilancia sobre la viabilidad de los recursos naturales que dan soporte a las necesidades sociales.
\end{abstract}

Palabras clave: Cambio climático. México. Gobernación. Sustentabilidad socio-ambiental.

\section{GOVERNANCE OF CLIMATE CHANGE IN MEXICO: PROSPECTS FOR REFORMS AT SUB-NATIONAL LEVEL}

\begin{abstract}
This article reviews the governance of climate change in Mexico, and the prospects for reforms at sub-national level. Following international orientation, the federal government has taken on the challenge of dealing with climate variability, in the belief that can, on the one hand, guide the developing world; and on the other, promote reforms to the public administration that are of high impact on sub-national demarcations. The central government has addressed a comprehensive strategy that revolves around a system of actions against climate change. However, the complexity of the problem surpasses centralist approaches,

\footnotetext{
${ }^{1}$ Doctor en Política y Sociología (Universidad Carlos III-Madrid). Afiliación institucional: ProfesorInvestigador. Dpto. Estudios Urbanos y del Medio Ambiente. El Colegio de la Frontera Norte. km 18.5 carr. esc. Tijuana-Ensenada. Tijuana, B.C. 22560, MEXICO. Correo electrónico: ricardosantes@gmail.com, rsantes@colef.mx. URL: http://www.colef.mx
}

DRd - Desenvolvimento Regional em debate (ISSNe 2237-9029) 
calling for the participation of state and municipal governments, whose institutional capacities are heterogeneous and must be strengthened to generate performances and intergovernmental arrangements that are efficient. A qualitative analysis of legal documents and policy instruments reveals the need of reforming the governance of climate change in Mexico. In this perspective, attention to the phenomenon demands public policy with a vision of integrity; also, consideration of two pendant tasks: decentralization and prioritization of workings towards vulnerable regions. In the interest of meeting the objectives of social and environmental sustainability, Mexico is compelled to build a scheme of governance that promotes intergovernmental and intersectoral coordination, broad participation of stakeholders, and alertness on the viability of the natural resources that maintain the social needs.

Keywords: Climate change. Mexico. Governance. Socio-environmental sustainability.

\section{INTRODUCCIÓN}

El cambio climático (CC) reclama atención de la comunidad internacional por los efectos actuales y potenciales que conlleva, los cuales alteran el equilibrio ecosistémico del planeta. México posee una visión empática con la creciente inquietud mundial, de manera que la vigilancia del fenómeno ha sido impulsada desde el propio gobierno central. Por las características físicas del país, la variación climática no repercute espacialmente de manera similar en el territorio: su impacto es mayor en algunas regiones (ocasionalmente con resultados funestos), amén de que los efectos se avizoran en períodos amplios. Tal vez por eso, los gobiernos estatales y municipales han mantenido actitudes diferenciadas frente al fenómeno de mayor o menor preocupación y ocupación. En ello, es cada vez más palpable que la intervención cuasi exclusiva del gobierno central es insuficiente, aun cuando reciba apoyo de instancias externas. La problemática que representa el CC para su correcta atención pública no admite actitudes evasivas de los gobiernos sub-nacionales. Ellos son actores principales en la inercia del crecimiento poblacional, la diversificación de usos de suelos, la urbanización, y la celeridad de las actividades productivas acompañantes. En esto, el uso y abuso de la naturaleza y sus recursos es motivo de alerta, pues pese a que la dinámica socioeconómica sirve a los propósitos del bienestar, el empleo irreflexivo de recursos naturales no sólo atenta contra la sustentabilidad ecosistémica, sino que es factor coadyuvante para la modificación del clima y de los graves impactos sociales que ya ocurren en algunas regiones.

Arguyendo que una visión de integralidad de la política pública es inseparable de los objetivos de sustentabilidad social y ambiental, en este trabajo se revisa la evolución de la política de CC de México y las expectativas de reforma a la gobernación del fenómeno en el escenario sub-nacional. Ello, mediante el análisis cualitativo de documentos normativos e instrumentos de política pública, como leyes, planes y programas. Los datos se obtuvieron tanto en bibliotecas como en sitios de internet oficiales de dependencias federales y estatales. Se buscó, por una parte, determinar la relevancia de la legislación federal relacionada con el tema ambiental en general y el cambio climático en particular; por otra, descubrir las características de la propuesta de acción gubernamental. En adición, se pretendió develar 
avances en la conformación de una política de acción frente al cambio climático por parte de los gobiernos locales, sea por creación de regulaciones o de programas específicos.

En la siguiente sección del artículo, se expone un panorama del escenario internacional alrededor del CC, que comprende los acuerdos y directivas generados a lo largo del tiempo. Lo anterior, porque el debate internacional se torna en el eje orientador de la experiencia mexicana. En la tercera parte, se destaca el desempeño de México y las reformas para la gobernación del fenómeno climático impulsadas por la administración federal a través de una estrategia nacional. En la cuarta sección, se examina el arreglo institucional subnacional, señalando la importancia de fortalecerlo a través de esquemas de planeación y gestión que incrementen la participación amplia, la coordinación intergubernamental e intersectorial, y que además contemplen la viabilidad de la base de recursos que soportan las necesidades sociales. Finalmente, se presentan reflexiones sobre avances y tareas pendientes de México para el logro de una mejor gobernación del CC.

\section{CAMBIO CLIMÁTICO: UNA PREOCUPACIÓN GLOBAL}

\section{PROBLEMAS DE DEFINICIÓN Y AMENAZAS REALES}

El Panel Intergubernamental sobre Cambio Climático (PICC) y la Convención Marco de las Naciones Unidas sobre Cambio Climático (CMNUCC) definen el fenómeno de manera distinta. Para el primero, se trata de una modificación climática que persiste durante un período prolongado, de décadas o más tiempo, debido a la variabilidad natural o como resultado de la actividad humana; para la segunda, consiste en una alteración atribuible directa o indirectamente a la actividad humana que modifica la composición atmosférica y que se suma a la mutabilidad natural detectada en períodos de tiempo comparables (NACIONES UNIDAS, 1992, p. 6; IPCC, 2007, p. 30). La diferencia no es sólo un matiz; exhibe el carácter político del tema, o la pretensión de insinuar causales de modo menos dispar, entre humanas y naturales. Aunque es incontrovertible que la anormalidad tiene existencia sustentada en evidencia científica y que, conforme ésta se acumula, confirma que la acción humana tiene la incidencia principal.

Hoy se argumenta que "El negacionismo del cambio climático de origen antropógeno ha perdido definitivamente la batalla" (SEMARNAT, 2012a, p. 20); las actividades humanas catalizan el fenómeno de manera inusitada. La manifestación más evidente es el calentamiento global, que ocurre por la alta concentración en la atmósfera de gases de efecto invernadero (GEI) resultantes de acciones antropogénicas como uso de combustibles, tala y quema de bosques, prácticas agrícolas y canje de uso de suelo. Los GEI liberados a la atmósfera atrapan más energía del sol y causan el calentamiento planetario; en ese sentido, la acción humana suscita el deshielo de los casquetes polares y el retroceso de los glaciares, el incremento del nivel del mar, el aumento en la temperatura media global, la alteración en los regímenes de precipitación, la aparición de plagas y enfermedades, y el aumento en las frecuencias e intensidades de eventos climáticos extremos (IPCC, 2007). 
Desde la primera Conferencia Mundial sobre el Clima, en 1979, se advirtió sobre la injerencia humana en el asunto (UNFCCC, 2007, p. 11). Consecuentemente, desde su creación en 1988 el PICC tuvo el mandato de analizar la información sobre transformaciones en las condiciones climáticas provocadas por las actividades humanas y las posibilidades de acomodación a las mismas o su atenuación. El Panel avizora para el continente americano una serie de riesgos durante los primeros 100 años del siglo. Advierte que la temperatura media de la superficie terrestre aumentará entre $1.8^{\circ} \mathrm{C}$ y $4.0^{\circ} \mathrm{C}$; que disminuirá el agua del suelo, ocurrirá una pérdida significativa de biodiversidad, un decrecimiento en la productividad de cosechas y la ganadería, y se presentará afectación en la disponibilidad de agua para consumo humano, agricultura y generación de energía; asimismo, que el impacto será preocupante en regiones costeras (IPCC, 2007, p. 45, 50-52).

Se aduce que quienes sufren ya los efectos del $\mathrm{CC}$ han sido los menos responsables. El mapa interactivo de emisiones de $\mathrm{CO}_{2}$ en 186 países, que presenta el medio británico The Guardian (2011) es útil para el análisis del problema pues, con información de organizaciones como World Resource Institute CAIT v8, International Energy Agency, y PNAS.org, orienta la discusión; ahí se determina que, pese a la dificultad de señalar cargas inequívocamente, pues hay al menos ocho criterios de referencia para emisiones de carbón, el mundo desarrollado sobresale en la mayoría de las mediciones. En efecto, los países desarrollados, con el 15 por ciento de la población mundial, producen aproximadamente el 80 por ciento de las emisiones de GEI. Independientemente del enfoque, el CC es una preocupación mundial que ahora se atiende para, al menos, aminorarlo y plantear alternativas.

La CMNUCC reconoce que el fenómeno y sus efectos son negativos, que las actividades productivas que emiten dióxido de carbono y otros gases han aumentado las concentraciones e intensificado el efecto invernadero. Con esa idea, desde su entrada en vigor en 1994, pretendió estabilizar las concentraciones de GEI a un nivel que, a la vez que impidiera interferencias antropogénicas peligrosas, fuera asequible en un plazo que permitiera la adaptación natural, la producción segura de alimentos, y el curso sostenible del crecimiento económico (IPCC, 2012).

La CMNUCC defiende la equidad, las capacidades y las responsabilidades comunes pero diferenciadas, y destaca las condiciones específicas de los miembros (Partes) en vías de desarrollo. Asumiendo las diferencias entre países se distingue también su contribución desigual al CC, lo que determina tres categorías y obligaciones de Partes: 1) desarrolladas; 2) en transición hacia una economía de mercado; y 3) en desarrollo. Los miembros se distribuyeron en anexos: 41 (entre industrializados y otros desarrollados, así como en proceso de transición a una economía de mercado) quedaron incluidos en el Anexo I; y de esa lista, 24 (industrializados y otros desarrollados) se incluyeron igualmente en el Anexo II. Este último grupo corresponde al subconjunto de países del Anexo I que en 1992 integraban la Organización para la Cooperación y el Desarrollo Económicos, quienes asumieron el compromiso de coadyuvar a que las Partes en desarrollo cumplieran con sus obligaciones, a través de apoyos financieros (SEMARNAT, 2012a, p. 28).

En conformidad con la Convención, todos los miembros consumarían compromisos generales y específicos relativos al Anexo correspondiente (NACIONES UNIDAS, 1992). En lo sustancial, los gobiernos se comprometieron a producir información sobre emisiones de GEI, políticas nacionales y mejores prácticas; instrumentar medidas nacionales respecto a dichas emisiones; generar esquemas de acomodación a efectos previsibles; y apoyar a países 
en desarrollo. Las estrategias principales serían la adaptación o reducción de la vulnerabilidad, y la atenuación o reducción de los GEI.

\section{DE KIOTO AL GRAN ACUERDO}

Siguiendo a la CMNUCC, en 1997 se adoptó el texto del Protocolo de Kioto (NACIONES UNIDAS, 1998), en el cual se establecen compromisos del Anexo I para reducir el total de emisiones de seis gases, que influyen en el calentamiento global, a un nivel inferior en no menos de 5 por ciento al de 1990 en el período 2008-2012. Logros notables son el acuerdo de una respuesta global al problema, la generación de políticas nacionales, la creación de un mercado internacional del carbono y el establecimiento de mecanismos institucionales que apoyarían futuros esfuerzos de mitigación (IPCC, 2007, p. 62).

Las reuniones de Bali, Copenhague, Cancún, Durban y Doha, que ocurrieron entre 2007 y 2012, revelan la permanente preocupación internacional por la anormalidad climática; sin embargo, los avances no han sido los esperados. Los 195 países que han ratificado la CMNUCC (SEMARNAT, 2012a, p. 27) convinieron que en 2009 se tendría un nuevo pacto que sustituiría a Kioto y entraría en vigor en 2013. Nada sucedió, de manera que ahora se vive una prórroga del protocolo. Un rasgo importante es que, por vía de la Plataforma de Durban para la Acción Ampliada, hacia 2015 el formato de Kioto cambiará, es decir, el régimen climático donde solamente los países desarrollados se obligan a reducir emisiones abre paso a uno en el que todas las partes, desarrolladas y en vías de desarrollo, asumirán compromisos jurídicamente vinculantes a partir de 2020 (SEMARNAT, 2013, p. 12).

Se trata de lo que algunos denominan el Gran Acuerdo; resta ver si los países, sobre todo aquellos que generan las mayores emisiones, cumplen con ese propósito o dejan constancia que el acuerdo no es más que otro malabarismo para continuar retrasando medidas que contengan la problemática. Lo anterior porque, como se comenta en medios (MÉNDEZ, 2012), "Los gigantes en desarrollo [...] temen que les impongan un sistema de verificación de sus emisiones que destripe su economía"; además demandan objetivos de reducción de emisiones para los desarrollados, especialmente Estados Unidos. Con todo, Washington permanecerá inamovible si no hay propósitos que obliguen a China e India. El problema estriba, simplemente, en que las emisiones de $\mathrm{CO}_{2}$ están ligadas a sectores estratégicos, como industria, transporte, y agricultura; cualquier iniciativa de CC impacta el crecimiento de áreas de la economía que utilizan combustibles (prácticamente, toda actividad humana), por lo que el asunto se ha convertido en uno de los problemas de cooperación internacional más delicados (ANTAL, 2010, p. 102) y la única solución que muchos avizoran es prorrogar el statu quo y acaso firmar el Gran Acuerdo (MÉNDEZ, 2012; HEDEGAARD, 2012). 


\title{
DESEMPEÑO DE MÉXICO
}

\author{
ACTUACIÓN INTERNACIONAL
}

En todas las modalidades que presenta el mapa de The Guardian, México tiene un aporte reducido de emisiones de GEI respecto al total. Hacia 2006 su contribución representaba \pm 1.6 por ciento de un total de 715 millones de toneladas de $\mathrm{CO}_{2}$ (emisiones por quema de combustibles fósiles), mientras que en el período 2009-2011 disminuyó, oscilando entre 1.38 y 1.4 por ciento (GOB. DE MÉXICO, 2009, p. 5; THE GUARDIAN, 2011; SEMARNAT, 2013, p. 13). No obstante, como en la mayoría de los asuntos que se discuten en la arena global, México se ha mostrado protagonista en iniciativas de mejora; es así que en 1993, en la condición de país en desarrollo, ratificó la CMNUCC (GARCÍA, 2008, p. 1-2).

No estando incluido en el Anexo I, el país no se obligaba a satisfacer metas cuantitativas de reducción de emisiones, por lo que sus acciones representaban iniciativas voluntarias (GOB. DE GUANAJUATO, 2011, p. 19). Con todo, al ratificar la Convención concedió en satisfacer los compromisos previstos para países de ese Anexo. De tal forma, México se impuso la adopción de políticas y toma de medidas de atenuación, limitando las emisiones antropogénicas, así como protegiendo y mejorando los sumideros y depósitos de GEI. Adicionalmente, se comprometió a la aplicación de tales políticas y medidas conjuntamente con otras Partes.

Conforme a la CMNUCC (Artículo 4), los compromisos adquiridos son los siguientes (NACIONES UNIDAS, 1992, p. 5-7): 1) Generar inventarios de emisiones antropogénicas por fuentes y de absorción por sumideros de los GEI; 2) Formular y aplicar programas nacionales, e inclusive programas regionales, para mitigar [los efectos del] CC; 3) Promover y cooperar en la difusión de tecnologías, prácticas y procesos que reduzcan o prevengan emisiones antropogénicas de GEI; 4) Promover la gestión sostenible, la conservación y el reforzamiento de los sumideros y depósitos de GEI; 5) Cooperar en la preparación y adaptación de los impactos del CC; 6) Incorporar el $\mathrm{CC}$ a sus políticas nacionales en una concepción de atención a la salud y el cuidado ambiental; 7) Coadyuvar al incremento del conocimiento sobre el CC y apoyar al intercambio de información; 8) Fomentar la capacitación, concientización y participación pública; 9) Mantener comunicación actualizada con la Conferencia de las Partes.

Similarmente, sin encontrarse entre los países a quienes se dirige el Protocolo de Kioto, aunque siendo firmante desde 1998, México se comprometió a realizar acciones consecuentes. De acuerdo con el Protocolo, las Partes, entre otras cosas, y "teniendo en cuenta sus responsabilidades comunes pero diferenciadas y las prioridades, objetivos y circunstancias concretos de su desarrollo nacional y regional" formularán y mantendrán actualizados programas que contendrán medidas para mitigar, y facilitar la adaptación, al CC; los programas deben abordar los sectores de energía, transporte, industria, agricultura, silvicultura, y gestión de desechos (NACIONES UNIDAS, 1998, p. 10-11). En ese contexto, el país participa como Estado tercero en los Mecanismos de Desarrollo Limpio, que son instrumentos de mercado para incentivar medidas de reducción de emisiones de GEI. Con ellos, países desarrollados pueden financiar proyectos de mitigación de emisiones de países en 
desarrollo, en permuta por Certificados de Reducción de Emisiones abonables a su propio compromiso de reducción (NACIONES UNIDAS, 1998, p. 13; GOB. DEL DISTRITO FEDERAL, 2008, p. 42).

Es notable que, pese a su reducido aporte de GEI, México haya sido activo firmante de acuerdos internacionales e impulsor de estudios y reformas domésticas para enfrentar el problema. En 1996 publicó el Estudio de país: México ante el Cambio Climático; luego, en 1997, 2001, 2006, 2009 y 2012 ha presentado sendas Comunicaciones Nacionales, manifestando el compromiso de reducir sus emisiones de gases de efecto invernadero de aquí al 2050 (DEL VILLAR, 2011, p. vi; IBARRARÁN, BOYD, and MORENO, 2011, p. 223; SEMARNAT, 2012b). Puede suponerse que, ante la perspectiva de concretar el Gran Acuerdo en 2020, las medidas tomadas encontrarán al país con notas positivas en adelanto técnicocientífico, jurídico, organizacional, y político.

\section{ARREGLO INSTITUCIONAL: LEYES E INSTRUMENTOS DE POLÍTICA}

Como resultado de la asignación de atribuciones, esbozadas en leyes secundarias, diferentes órdenes de gobierno tienen incidencia en la problemática ambiental; inclusive en el tema del CC pese a que no se haga referencia específica necesariamente. En el cuadro 1 se resumen las leyes secundarias mexicanas con incidencia en el asunto.

Cuadro 1. Leyes federales relacionadas con el tema ambiental

\begin{tabular}{|l|l|}
\hline \multicolumn{1}{|c|}{ Ley federal } & \multicolumn{1}{|c|}{ Propósito } \\
\hline $\begin{array}{l}\text { Ley General de Bienes } \\
\text { Nacionales }\end{array}$ & Explicita los bienes sujetos al dominio público de la Federación \\
\hline Ley de Planeación & $\begin{array}{l}\text { Finca las bases de la planeación nacional del desarrollo, encauzando la } \\
\text { Administración Pública Federal (APF) en el Sistema de Planeación } \\
\text { Democrática }\end{array}$ \\
\hline $\begin{array}{l}\text { Ley Orgánica de la } \\
\text { Administración Pública } \\
\text { Federal }\end{array}$ & $\begin{array}{l}\text { Establece los fundamentos de organización de la APF, centralizada y } \\
\text { paraestatal }\end{array}$ \\
\hline $\begin{array}{l}\text { Ley General del Equilibrio } \\
\text { Ecológico y la Protección al }\end{array}$ & $\begin{array}{l}\text { Propicia el desarrollo sustentable, estableciendo bases para garantizar el } \\
\text { derecho a un ambiente adecuado y sustentable; define principios de política e } \\
\text { instrumentos ambientales; asegura la participación, la concurrencia } \\
\text { intergubernamental y entre autoridades y los sectores social y privado }\end{array}$ \\
\hline $\begin{array}{l}\text { Ley de Desarrollo Rural } \\
\text { Sustentable }\end{array}$ & $\begin{array}{l}\text { Promover el desarrollo rural sustentable, propiciar un medio ambiente } \\
\text { adecuado, y garantizar la rectoría del Estado en la promoción de la equidad }\end{array}$ \\
\hline $\begin{array}{l}\text { Ley de Desarrollo Sustentable } \\
\text { de la Caña de Azúcar }\end{array}$ & $\begin{array}{l}\text { Normar las actividades asociadas a la agricultura de contrato y a todas las } \\
\text { actividades agro-productivas inherentes a la caña de azúcar, hasta su etapa de } \\
\text { comercialización y la de sus productos, subproductos, coproductos y derivados }\end{array}$ \\
\hline $\begin{array}{l}\text { Ley de Promoción y } \\
\text { Desarrollo de los } \\
\text { Bioenergéticos }\end{array}$ & $\begin{array}{l}\text { Promover el desarrollo de los Bioenergéticos con el fin de coadyuvar a la } \\
\text { diversificación energética y el desarrollo sustentable, para apoyar al campo }\end{array}$ \\
\hline $\begin{array}{l}\text { Ley General de Desarrollo } \\
\text { Forestal Sustentable }\end{array}$ & $\begin{array}{l}\text { Regular y fomentar la conservación, protección, restauración, producción, } \\
\text { ordenación, el cultivo, manejo y aprovechamiento de los ecosistemas forestales } \\
\text { y sus recursos, así como distribuir las competencias correspondientes a la }\end{array}$ \\
\hline
\end{tabular}

DRd - Desenvolvimento Regional em debate (ISSNe 2237-9029) 


\begin{tabular}{|l|l|}
\hline & Federación, los Estados, el Distrito Federal y los Municipios \\
\hline $\begin{array}{l}\text { Ley General de Pesca y } \\
\text { Acuacultura Sustentables }\end{array}$ & $\begin{array}{l}\text { Regular, fomentar y administrar el aprovechamiento de los recursos pesqueros } \\
\text { y acuícolas en el territorio nacional y las zonas sobre las que la nación ejerce } \\
\text { su soberanía y jurisdicción }\end{array}$ \\
\hline $\begin{array}{l}\text { Ley para el Aprovechamiento } \\
\text { Sustentable de la Energía }\end{array}$ & $\begin{array}{l}\text { Propiciar un aprovechamiento sustentable de la energía mediante el uso óptimo } \\
\text { de la misma en todos sus procesos y actividades, desde su explotación hasta su } \\
\text { consumo }\end{array}$ \\
\hline Ley General de Vida Silvestre & $\begin{array}{l}\text { Establecer la concurrencia de los tres órdenes de gobierno, en el ámbito de sus } \\
\text { competencias, relativa a la conservación y aprovechamiento sustentable de la } \\
\text { vida silvestre y su hábitat en el territorio nacional y en las zonas en donde la } \\
\text { Nación ejerce su jurisdicción }\end{array}$ \\
\hline
\end{tabular}

Elaboración propia, con datos de la fuente: Gob. de México (2005; 2008a; 2008b; 2011; 2012b; 2012d; 2012e; 2013a; 2013c, 2014a, 2014b)

No es sencillo definir cómo una administración pública sectorizada ha de abordar el tema ambiental, que por su naturaleza desconoce límites geográficos, temáticos, o de gestión pública. Dado que diferentes órdenes de gobierno están obligados a atender los bienes nacionales, la Carta Magna faculta al Congreso para expedir leyes que establezcan la concurrencia del Gobierno Federal y los gobiernos sub-nacionales, en el ámbito de sus respectivas competencias en asuntos de protección al ambiente y de preservación y restauración del equilibrio ecológico.

En materia de $\mathrm{CC}$, luego de casi una década de avance legislativo lento en el nuevo siglo, México ha tenido logros importantes. Carrillo y Alanís $(2011$, p. 1.6) señalan que la celebración en 2010 de la $16^{\text {a }}$ Conferencia de las Partes de la CMNUCC, en Cancún, fue detonante de ese progreso. A partir de 2011 la LGEEPA estableció que la formulación y ejecución de acciones de mitigación y adaptación al CC son elementos de utilidad pública, y que en estos encargos tienen competencia tanto la Federación como los gobiernos de estados y municipios, de conformidad con lo dispuesto por la ley federal y las leyes locales. En consecuencia, la misma ley prevé los mecanismos para que la Federación, por conducto de la Secretaría de Medio Ambiente y Recursos Naturales, o Semarnat, suscriba convenios o acuerdos de coordinación, con los distintos órdenes de gobierno para que asuman, en el ámbito de su jurisdicción territorial, labores de administración, vigilancia, prevención, protección, restauración, control, y evaluación de impacto, entre otras, respecto a asuntos como: áreas naturales protegidas; suelo, flora, fauna y recursos forestales; zona federal marítimo terrestre y cuerpos de agua nacionales; y contaminación.

\section{Ley General de Cambio Climático}

Desde junio de 2012, México cuenta con la Ley General de Cambio Climático, o LGCC, la que de inmediato se ha convertido en el principal instrumento de política pública para atender el fenómeno (SEMARNAT, 2013, p. 13). La LGCC se propone garantizar el derecho a un ambiente sano; establecer la concurrencia de facultades entre órdenes de gobierno en la elaboración y aplicación de políticas públicas; regular las emisiones, lo mismo 
que las acciones para la mitigación y adaptación; reducir la vulnerabilidad humana y ecosistémica frente a los efectos adversos, así como crear y fortalecer las capacidades nacionales de respuesta; fomentar la educación, investigación, mejora y transferencia de tecnología e innovación y difusión; establecer las bases para la concertación con la sociedad; y promover la transición hacia una economía competitiva, sustentable y de bajas emisiones de carbono (GOB. DE MÉXICO, 2012c). Sobresale el Título Tercero, donde se indica la creación del Instituto Nacional de Ecología y Cambio Climático (INECC) que, al sustituir al Instituto Nacional de Ecología adiciona a su competencia el reto de la variación climática. La LGCC entró en vigencia en octubre de 2012 y sus propósitos se reflejan en las políticas del gobierno federal, que se exponen y defienden en el plan nacional.

Plan Nacional de Desarrollo

La reflexión sobre el CC ha sido relevante en el Plan Nacional de Desarrollo (PND); sobre todo durante las administraciones pasada y actual. El PND del período 2007-2012 tuvo como principio rector el desarrollo sustentable (GOB. DE MÉXICO, 2007, p. 23), planteando que éste exige reconocer que la estabilidad climática representa un bien, por lo que es primordial que México se sume a los esfuerzos internacionales por evitar dislocaciones ambientales planetarias, como el calentamiento global. Concentró las acciones en el fomento a la eficiencia en generación y uso de energía, el freno a la deforestación, y la reducción de emisiones de otros GEI (GOB. DE MÉXICO, 2007, p. 259). Asimismo, defendió la transversalidad de las políticas, considerándola como imprescindible en la integración intersectorial y la coordinación intergubernamental, con lo que dio por entendido que el desarrollo humano sustentable no corresponde a lo ambiental únicamente sino a todas las políticas sectoriales. En esa línea, el PND indicó el diseño de las políticas relacionadas con la sustentabilidad ambiental en acuerdo con las dependencias de la APF y los tres órdenes de gobierno (GOB. DE MÉXICO, 2007, p. 27, 37, 234, 256).

El PND 2013-2018 ratifica al CC como tema principal. En el apartado I, México en Paz, en la sección sobre protección civil y prevención de desastres, se le presenta como un peligro para el bienestar, patrimonio y progreso de la población, así como los bienes públicos. Mientras que en los apartados IV y V (México Próspero y México con Responsabilidad Global, respectivamente), se subraya que frente a los efectos percibidos durante la última década el país ha asumido el compromiso de liderar el tema del CC en la agenda internacional (GOB. DE MÉXICO, 2013b, p. 37, 77, 91-92).

En el apartado VI, donde se desglosan los objetivos, estrategias y líneas de acción, el PND 2013-2018 trata sistemáticamente el CC. Para los propósitos de un México próspero, el Objetivo 4.4 plantea promover un crecimiento "verde" con tintes de inclusión y preservación del patrimonio natural. Aquí se destacan dos estrategias: 4.4.1 y 4.4.3. La primera consiste en avanzar una política de desarrollo que enlace la sustentabilidad ambiental con los costos y beneficios sociales; presenta nueve líneas de acción, donde sobresalen ideas como coordinación de programas gubernamentales, actualización de legislación ambiental, incremento en recursos para la protección ambiental, planeación territorial y, particularmente, construcción de una política de mares y costas que suscite "oportunidades económicas, 
fomente la competitividad, la coordinación" y, atendiendo a la defensa de bienes y servicios ambientales, enfrente los efectos del CC. La segunda estrategia del PND 2013-2018 pretende fortalecer la política de CC y cuidado ambiental "para transitar hacia una economía competitiva, sustentable, resiliente y de bajo carbono", y presenta 11 líneas de acción donde destacan propósitos como perfección de instituciones e instrumentos de política del sistema nacional de $\mathrm{CC}$; consolidación de la cooperación regional e internacional, biodiversidad y medio ambiente; investigación científica y tecnológica, generación y sistematización de información sobre políticas ambientales y de mitigación y adaptación al $\mathrm{CC}$; y consecución del ordenamiento ecológico territorial, especialmente en zonas de mayor vulnerabilidad climática (GOB. DE MÉXICO, 2013b, p. 134-135).

Para el mantenimiento de un México con responsabilidad global, el Objetivo 5.1 sugiere ratificar el protagonismo internacional. Se mencionan aquí dos estrategias relativas. Una (5.1.4), que pugna por consolidar la región Asia-Pacífico como toral para diversificar la economía mexicana; propone siete líneas de acción, donde sugiere la pertinencia de descubrir temas comunes, como el combate al CC. Otra (5.1.6) reitera la propuesta de consolidar al país como un actor principal en el ámbito multilateral; de ocho líneas de acción, se cita la de participar en la deliberación internacional sobre regímenes jurídicos, en tópicos de CC y otros de similar relevancia (GOB. DE MÉXICO, 2013b, p. 148-149).

Estrategia Nacional de Cambio Climático

La política nacional de CC se formula y conduce de acuerdo con 10 principios: 1) Sustentabilidad ambiental; 2) Corresponsabilidad Estado-Sociedad; 3) Precaución ante la incertidumbre; 4) Prevención de daños ambientales y preservación del equilibrio ecológico; 5) Adopción de patrones de producción y consumo sustentables; 6) Transversalidad e integralidad tanto intergubernamental como entre los sectores social, público y privado; 7) Participación ciudadana; 8) Responsabilidad; 9) Transparencia, acceso a la información y a la justicia; 10) Crecimiento económico sin comprometer la competitividad frente a los mercados internacionales (SEMARNAT, 2013, p. 17). Tales principios son los referentes que permiten la construcción de la Estrategia Nacional de Cambio Climático (ENCC).

El trabajo de las burocracias que pretenden contrarrestar los impactos del $\mathrm{CC}$ ha evolucionado con cierta celeridad; máxime por la premura de encarar un fenómeno juzgado como "problema de seguridad estratégica nacional (y mundial)" (GARCÍA, 2008, p. 3; CICC, 2006 , p. 3). Desde su publicación por primera vez, en 2000, girando alrededor de las metas de mitigación y adaptación, la ENCC definió tres objetivos importantes: 1) Consolidar normas, políticas y programas en torno a las emisiones de GEI; 2) Convocar a la corresponsabilidad entre gobierno y sociedad para mejorar prácticas de uso y consumo bajo una perspectiva ambientalmente compatible; 3) Orientar a los diversos actores de la administración pública y la vida nacional en general, a planear y realizar acciones de mitigación y adaptación (SEMARNAP, 2000, p. 35).

La LGCC (Artículo 60) considera a la ENCC como el instrumento que rige la política de mediano y largo plazos para afrontar el fenómeno y "transitar hacia una economía competitiva, sustentable y de bajas emisiones de carbono" (GOB. DE MÉXICO, 2012c). En

DRd - Desenvolvimento Regional em debate (ISSNe 2237-9029) v. 5, n. 1, p. 88-110, jan./jun. 2015. 
ese sentido, la ENCC plantea prioridades nacionales y define criterios para identificar las de escala regional. En forma optimista plantea que en el largo plazo México "crecerá de manera sostenible y promoverá el manejo sustentable y equitativo de sus recursos naturales, así como el uso de energías limpias y renovables"; supone por tanto, armonía entre crecimiento y ambiente. Conjetura igualmente que el país se tornará "próspero, competitivo, socialmente incluyente y con responsabilidad global, generando empleos suficientes y bien remunerados para toda su población, en particular para la más vulnerable”. Propone de esta forma un México con equidad social, economía verde y ciudades sustentables, así como con “ecosistemas y poblaciones resilientes" al CC (SEMARNAT, 2013, p. 9, 22).

Con todo, la ENCC recibió críticas en el aspecto de mitigación por no contar con objetivos claros o metas de reducción específicas; también, por carecer de líneas de acción concretas y mecanismos para avanzar sus objetivos (IBARRARÁN, BOYD, and MORENO, 2011, p. 224). Seguramente por esa razón el gobierno federal impulsó la creación del Sistema Nacional de Acción frente al Cambio Climático (Sinacc), con el cual propone conducir acciones conjuntas para enfrentar la vulnerabilidad y los riesgos del país y establecer las medidas de mitigación y adaptación pertinentes. El Sinacc se conforma por instancias como: Comisión Intersecretarial de Cambio Climático, Consejo de Cambio Climático (CCC), INECC, entidades federativas, autoridades municipales, y Congreso de la Unión (SEMARNAT, 2013, p. 13).

La Comisión Intersecretarial de Cambio Climático (CICC) constituye el órgano permanente de coordinación entre las dependencias y entidades de la APF en la materia; formula políticas nacionales de mitigación y adaptación, y procura su incorporación a programas y acciones sectoriales; asimismo, genera criterios de transversalidad e integralidad en dependencias y entidades de la APF centralizada y paraestatal; también se encarga de aprobar la ENCC y participa en la elaboración e instrumentación del Programa Especial de Cambio Climático, o PECC (SEMARNAT, 2013, p. 14). La CICC se creó en 2005 con la participación de los representantes de siete secretarías (Relaciones Exteriores; Desarrollo Social; Medio Ambiente y Recursos Naturales; Energía; Economía; Agricultura, Ganadería, Desarrollo Rural, Pesca y Alimentación, y Comunicaciones y Transportes). En la actualidad se han incorporado otras seis dependencias federales para conformar un cuerpo plural y multitemático, a saber: Gobernación, Marina, Hacienda y Crédito Público, Educación Pública, Salud, y Turismo (GOB. DE MÉXICO, 2005; SEMARNAT, 2013, p. 14). Por su parte, el CCC es la instancia de consulta permanente de la CICC; se conforma por representantes de los sectores social, privado y académico. Funciones relevantes son las de asesoría a la CICC (sugiriendo la realización de estudios, políticas y acciones, así como el establecimiento de metas) y la promoción de una participación social informada (SEMARNAT, 2013, p. 14).

El Sinacc posee una estructura de tres grandes componentes: 1) Pilares, que definen las metas o grandes objetivos de la política nacional; 2) Ejes Estratégicos, que se centran en objetivos concretos; y 3) Líneas de Acción, que son rutas que demandan participación, integración y continuidad, además que señalan aspectos importantes para su consecución. El Sinacc se sustenta en seis pilares y ocho ejes estratégicos; de estos últimos, tres se enfocan al objetivo de adaptación y cinco al de mitigación. Por su parte, de 199 líneas de acción que se enuncian en el sistema, 83 corresponden a los pilares y 116 a los ejes (41 en el rubro de adaptación y 75 en el de mitigación). El Cuadro 2 sintetiza la estructura del Sinacc. 
Cuadro 2. Pilares, ejes estratégicos y número de líneas de acción que sustentan el Sinacc

\begin{tabular}{|c|c|}
\hline Pilares para la construcción de la política $(\mathrm{P})$ & No. de líneas \\
\hline $\begin{array}{l}\text { P1. Contar con políticas y acciones climáticas transversales, articuladas, coordinadas e } \\
\text { incluyentes }\end{array}$ & 18 \\
\hline $\begin{array}{l}\text { P2. Desarrollar políticas fiscales e instrumentos económicos y financieros con enfoque } \\
\text { climático }\end{array}$ & 18 \\
\hline $\begin{array}{l}\text { P3. Implementar una plataforma de investigación, innovación, desarrollo y adecuación de } \\
\text { tecnologías climáticas y fortalecimiento de capacidades institucionales }\end{array}$ & 20 \\
\hline P4. Promover el desarrollo de una cultura climática & 7 \\
\hline P5. Instrumentar mecanismos de medición, reporte, verificación, y monitoreo y evaluación & 12 \\
\hline P6. Fortalecer la cooperación estratégica y el liderazgo internacional & 8 \\
\hline Subtotal & 83 \\
\hline Ejes estratégicos de adaptación al CC (A) & No. de líneas \\
\hline $\begin{array}{l}\text { A1. Reducir la vulnerabilidad y aumentar la resiliencia del sector social ante los efectos del } \\
\text { CC }\end{array}$ & 13 \\
\hline $\begin{array}{l}\text { A2. Reducir la vulnerabilidad y aumentar la resiliencia de la infraestructura estratégica y } \\
\text { sistemas productivos ante los efectos del CC }\end{array}$ & 12 \\
\hline $\begin{array}{l}\text { A3. Conservar y usar de forma sustentable los ecosistemas y mantener los servicios } \\
\text { ambientales que proveen }\end{array}$ & 16 \\
\hline Ejes estratégicos de mitigación del CC $(\mathrm{M})$ & No. de líneas \\
\hline M1. Acelerar la transición energética hacia fuentes de energía limpia & 14 \\
\hline $\begin{array}{l}\text { M2. Reducir la intensidad energética mediante esquemas de eficiencia y consumo } \\
\text { responsable }\end{array}$ & 14 \\
\hline $\begin{array}{l}\text { M3. Transitar a modelos de ciudades sustentables con sistemas de movilidad, gestión } \\
\text { integral de residuos y edificaciones con baja huella de carbono }\end{array}$ & 13 \\
\hline $\begin{array}{l}\text { M4. Impulsar mejores prácticas agropecuarias y forestales para incrementar y preservar los } \\
\text { sumideros naturales de carbono }\end{array}$ & 15 \\
\hline $\begin{array}{l}\text { M5. Reducir emisiones de contaminantes climáticos de vida corta y propiciar co-beneficios } \\
\text { de salud y bienestar }\end{array}$ & 19 \\
\hline Subtotal & 116 \\
\hline Total & 199 \\
\hline
\end{tabular}

Elaboración propia, adaptado de la fuente Semarnat (2013)

En concordancia con la ENCC, en agosto de 2009 se aprobó el primer Programa Especial de Cambio Climático (PECC) para el período 2009-2012; con ello, el gobierno federal intentó demostrar la factibilidad de aminorar el fenómeno y "adaptarse, sin comprometer el proceso de desarrollo, e incluso con beneficio económico" (GOB. DE MÉXICO, 2009; GOB. DE HIDALGO, 2011, p. 33). El PECC define los objetivos de la administración en turno y señala acciones sexenales específicas de mitigación y adaptación, identificando entidades encargadas y metas (SEMARNAT, 2013, p. 18). El segundo programa, PECC 2014-2018, vigente a partir de abril de este año, pretende consolidar el Sinacc pues, manteniendo la perspectiva nacional, busca afianzar la política en la materia en coordinación con entidades federativas, municipios, y Poder Legislativo, amén de que reitera el propósito de incorporar a la sociedad (GOB. DE MÉXICO, 2014c). 
Si bien la corresponsabilidad Estado-Sociedad se reconoce como un principio a la vez que un objetivo de la ENCC, el papel que se garantiza a la sociedad es menor. Baste con ver el peso que se da en el Sinacc a las instancias del Estado a través de la CICC y la condición de "asesoría" que se otorga a ciertos representantes del sector no gubernamental dentro del CCC. Si la corresponsabilidad queda entendida como "el reconocimiento y aceptación de las consecuencias de acciones u omisiones de los actores, así como de su diferencial de peso específico respecto a objetivos compartidos" (SANTES-ÁLVAREZ, 2009, p. 30), la influencia del sector social en la orientación de la política nacional de CC tendría que incrementarse sustancialmente. Para que ocurra un giro en las prácticas cotidianas, que coadyuven al éxito de la ENCC, se requiere de un marco de acuerdo gobierno-sociedad más que de una imposición; ello es factible si los actores de la sociedad y el Estado asumen el compromiso de conducirse conforme a sus responsabilidades comunes pero diferenciadas.

\section{GOBERNACIÓN DEL CAMBIO CLIMÁTICO EN ESTADOS Y MUNICIPIOS}

\section{MARCO NORMATIVO E INSTRUMENTOS DE POLÍTICA}

Hasta antes de 2012, la falta de una legislación federal específica no había causado inmovilidad legislativa en algunas de las 32 entidades mexicanas. Veracruz, Chiapas y el Distrito Federal fueron pioneras en generar su propia ley sobre CC. Con cierta lentitud, otras entidades han ido sumándose a un nuevo contexto institucional donde el fenómeno climático incide con mayor fuerza en la esfera pública. Esto ha ocurrido, esencialmente, luego de la creación de la LGCC, en junio de 2012. El Cuadro 3 recoge una cronología y los objetivos principales de las 14 leyes estatales vigentes.

Cuadro 3. Leyes estatales de cambio climático $(*)$

\begin{tabular}{|c|c|c|}
\hline Entidad federativa / Ley & $\begin{array}{l}\text { Fecha de } \\
\text { creación }\end{array}$ & Objetivos principales de las leyes \\
\hline $\begin{array}{l}\text { Veracruz: Ley estatal de } \\
\text { mitigación y adaptación ante } \\
\text { los efectos del CC }\end{array}$ & $\begin{array}{l}\text { Noviembre } \\
2010\end{array}$ & \multirow{5}{*}{$\begin{array}{l}\text { Política y administración públicas: } \\
\text { 1. Definir criterios de políticas de prevención y } \\
\text { adaptación, así como de cuidado ecosistémico } \\
\text { (políticas transversales) } \\
\text { 2. Formular, conducir y evaluar la política estatal } \\
\text { en la materia } \\
\text { 3. Desarrollar indicadores y evaluar resultados } \\
\text { 4. Promover la concurrencia estatal y municipal } \\
\text { 5. Establecer bases de coordinación } \\
\text { intergubernamental } \\
\text { 6. Instrumentar mecanismos de concertación } \\
\text { gobierno-sociedad, para la formulación e } \\
\text { instrumentación de políticas de adaptación y } \\
\text { mitigación } \\
\text { 7. Establecer bases para la acción de los sectores } \\
\text { social y privado } \\
\text { Planificación de acciones: } \\
\text { Unidades gubernamentales }\end{array}$} \\
\hline $\begin{array}{l}\text { Chiapas: Ley para la } \\
\text { adaptación y mitigación ante } \\
\text { el CC del Estado }\end{array}$ & $\begin{array}{l}\text { Diciembre } \\
2010 / \text { abril } \\
2013 \\
\text { (reforma } \\
\text { total) }\end{array}$ & \\
\hline $\begin{array}{l}\text { Distrito Federal: Ley de } \\
\text { mitigación y adaptación al } \\
\text { CC y desarrollo sustentable }\end{array}$ & Junio 2011 & \\
\hline $\begin{array}{l}\text { Baja California: Ley de } \\
\text { prevención, mitigación y } \\
\text { adaptación del CC para el } \\
\text { Estado }\end{array}$ & Junio 2012 & \\
\hline $\begin{array}{l}\text { Coahuila: Ley para la } \\
\text { Adaptación y Mitigación a } \\
\text { los efectos del Cambio } \\
\text { Climático en el Estado }\end{array}$ & Enero 2013 & \\
\hline
\end{tabular}

DRd - Desenvolvimento Regional em debate (ISSNe 2237-9029)

v. 5, n. 1, p. 88-110, jan./jun. 2015. 


\begin{tabular}{|c|c|c|}
\hline $\begin{array}{l}\text { Quintana Roo: Ley de Acción } \\
\text { de Cambio Climático en el } \\
\text { Estado }\end{array}$ & Abril 2013 & $\begin{array}{l}\text { 8. Conformar recursos instrumentales } \\
\text { (programas, proyectos) para avanzar las medidas } \\
\text { necesarias }\end{array}$ \\
\hline $\begin{array}{l}\text { Durango: Ley de Cambio } \\
\text { Climático del Estado }\end{array}$ & Mayo 2013 & $\begin{array}{l}\text { 9. Incentivar el fortalecimiento de las } \\
\text { capacidades institucionales y sectoriales }\end{array}$ \\
\hline $\begin{array}{l}\text { Chihuahua: Ley de Cambio } \\
\text { Climático del Estado }\end{array}$ & Junio 2013 & $\begin{array}{l}\text { 10. Instrumentar medidas de protección de la } \\
\text { población }\end{array}$ \\
\hline $\begin{array}{l}\text { Hidalgo: Ley de Mitigación y } \\
\text { Adaptación ante los efectos } \\
\text { del Cambio Climático para el } \\
\text { Estado }\end{array}$ & $\begin{array}{l}\text { Agosto } \\
2013\end{array}$ & $\begin{array}{l}\text { 11. Prevenir y controlar las emisiones que no } \\
\text { sean de competencia federal } \\
\text { Sector social } \\
\text { 12. Promover la participación social }\end{array}$ \\
\hline $\begin{array}{l}\text { Guanajuato: Ley de Cambio } \\
\text { Climático para el Estado de } \\
\text { Guanajuato y sus Municipios }\end{array}$ & $\begin{array}{l}\text { Noviembre } \\
2013\end{array}$ & $\begin{array}{l}\text { 13. Fomentar la educación y la generación de } \\
\text { conocimiento científico y tecnológico en materia } \\
\text { de adaptación y mitigación }\end{array}$ \\
\hline $\begin{array}{l}\text { Oaxaca: Ley de Cambio } \\
\text { Climático del Estado }\end{array}$ & $\begin{array}{l}\text { Noviembre } \\
2013\end{array}$ & $\begin{array}{l}\text { 14. Fomentar la creación de una cultura } \\
\text { preventiva, así como crear y fortalecer las }\end{array}$ \\
\hline $\begin{array}{l}\text { Puebla: Ley de Cambio } \\
\text { Climático del Estado }\end{array}$ & $\begin{array}{l}\text { Noviembre } \\
2013\end{array}$ & $\begin{array}{l}\text { capacidades de respuesta para disminuir la } \\
\text { vulnerabilidad }\end{array}$ \\
\hline $\begin{array}{l}\text { Estado de México: Ley de } \\
\text { Cambio Climático del Estado }\end{array}$ & $\begin{array}{l}\text { Diciembre } \\
2013\end{array}$ & $\begin{array}{l}\text { 15. Garantizar el derecho a un ambiente } \\
\text { adecuado }\end{array}$ \\
\hline $\begin{array}{l}\text { Michoacán: Ley de Cambio } \\
\text { Climático del Estado }\end{array}$ & Enero 2014 & $\begin{array}{l}\text { Sector productivo } \\
\text { 16. Promover la puesta en marcha de } \\
\text { instrumentos económicos para la mitigación y la } \\
\text { adaptación } \\
\text { 17. Garantizar, la eficiencia energética } \\
\text { 18. Establecer las bases para una economía verde } \\
\text { Medio ambiente } \\
\text { 19. Impulsar el manejo sustentable de los } \\
\text { recursos naturales } \\
\text { 20. Procurar la conservación de la biodiversidad }\end{array}$ \\
\hline
\end{tabular}

Elaboración propia, con apoyo en: ALDF (2011); Congresos estatales de Baja California (2012), Chiapas (2013), Chihuahua (2013), Coahuila (2013), Durango (2014), Guanajuato (2013), Hidalgo (2013), México (2013), Michoacán (2014), Oaxaca (2013), Puebla (2013), Quintana Roo (2013), y Veracruz (2013).

(*) Actualidad: mayo 2014

Las directivas legales y las estrategias de política de atención al CC se concretan en espacios determinados por vía de organizaciones burocráticas. Los gobiernos locales catalizan las acciones, contando por lo regular con los apoyos federales necesarios. En ese ánimo, desde la Federación se subraya la importancia de que todas las entidades cuenten con un Programa Estatal de Acción ante el Cambio Climático (PEACC) con el fin de "desarrollar capacidades institucionales que los hagan resilientes a los impactos de este fenómeno global" (SEMARNAT, 2011). Con el apoyo del INECC, los programas avanzan en los diferentes estados. Definidos como instrumentos para el diseño de políticas públicas sustentables y acciones específicas, además de elementos importantes para la política climática, los PEACC deben considerar las directivas de los planes estatales, así como las características sociales, económicas y ambientales de cada entidad. Igualmente, deben tomar en cuenta el inventario, los escenarios de emisiones de GEI, y la posible modificación climática estatal-regional, con las acciones de gestión para prevenirlos o reducirlos (INECC, 2013).

Como colaborador en la creación de los programas estatales, el INECC no tiene obligatoriedad en los trabajos de cada uno. Tal vez por ese motivo, en los 10 PEACC que hasta marzo de 2013 se declararon como concluidos (Distrito Federal en 2008; Veracruz en 2009; Nuevo León y Puebla en 2010; Chiapas, Guanajuato, Hidalgo, y Tabasco en 2011; Baja 
California en 2012; y Baja California Sur en 2013) se observan diferencias en estructura y profundidad; ciertamente, el énfasis respecto de algunos tópicos responde a prioridades de cada entidad. A los programas concluidos el INECC agregaba en su sitio de internet 16 que se hallaban en proceso de planeación o construcción (INECC, 2013); sin embargo, hacia 2014 no existe información actualizada, aunque parece factible que las entidades interesadas pronto presenten sus avances, en los que se esperaría que privilegien su propia realidad ambiental y sociocultural.

Otro instrumento que se gesta en México desde 2011 para capacitar y fortalecer a los gobiernos municipales en los esfuerzos para enfrentar el CC es el Plan de Acción Climática Municipal (Pacmun). Impulsado por ICLEI-Gobiernos Locales por la Sustentabilidad y el INECC, y recibiendo financiamiento de la Embajada Británica, el Pacmun tiene como objetivo procurar que los gobiernos municipales establezcan políticas para reducir emisiones de GEI y de otros contaminantes. Hasta inicios de 2014, el sitio de internet de ICLEI registraba un total de 242 municipios mexicanos participando en esta iniciativa; de ellos, 31 contaban con una programa acabado (ICLEI, 2014).

En cuanto a figuras organizacionales, algunas entidades destacan por haber creado instancias intersectoriales ad hoc, como las Comisiones Estatales Intersecretariales sobre CC (CEICC). Las existentes en 17 entidades (Campeche, Coahuila, Distrito Federal, Durango, Guanajuato, Guerrero, Jalisco, Michoacán, Querétaro, Quintana Roo, San Luis Potosí, Sinaloa, Tabasco, Tamaulipas, Tlaxcala, Veracruz y Yucatán) y otras que se gestan (en Hidalgo y Nayarit por ejemplo) constituyen alternativas para coordinar acciones entre las dependencias locales (IDLO, 2011, p. 16; LA RAZÓN, 2013). Pese a lo idóneo que sean esas instancias, su creación no dice mayor cosa, pues no ha transcurrido tiempo suficiente para evaluar su desempeño. Por otro lado, es factible que las adecuaciones en el orden estatal impacten el ámbito de la planeación de los municipios, pues los efectos del CC motivarán que instancias como los consejos de planeación del desarrollo y los institutos municipales/metropolitanos de planeación acuerden acciones para afrontar el fenómeno.

Del Villar afirma que estados y municipios muestran fuerte activismo en reacciones legales y de política pública, debido tanto a sus propias facultades como a las ventajas respecto de la Federación por "la inmediatez de su capacidad de respuesta ante los efectos del calentamiento global" (DEL VILLAR, 2011, p. vi). Leyes estatales, PEACC, Pacmun y CEICC pueden ser prueba de esta aseveración.

\section{APTITUDES INSTITUCIONALES}

Las capacidades institucionales de los gobiernos sub-nacionales son heterogéneas, con frecuencia acusando debilidades en recursos materiales y humanos. Los estados caminan a ritmos dispares en sus estrategias de acción frente al $\mathrm{CC}$; como se ha visto, algunos se sustentan en leyes locales de protección al ambiente, mientras que otros ya lo hacen en marcos jurídicos específicos, adelantándose a la Federación inclusive. A la vez, hay entidades que conforman estructuras organizacionales novedosas como las CEICC, con lo que muestran voluntad por resolver las problemáticas y mejorar la gobernación del CC en sus territorios, que se potencia con la perspectiva de los PEACC. 
Tales iniciativas contribuyen a los objetivos de la sustentabilidad y la calidad de vida de la población; no obstante, la inercia del embrollo burocrático y centralista obstaculiza la reforma de la gestión pública mexicana. Las administraciones locales están frente al reto de procurar visiones programáticas de largo plazo en cuanto a trayectorias de mitigación y adaptación; de similar manera, vigilar que los objetivos de la transversalidad de la política tengan eco en todos los sectores. En ese sentido, los gobiernos locales han de plantearse el trascender la visión monocéntrica de la gobernación, atada al supuesto de las escalas jurisdiccionales (MORENO PLATA, 2013, p. 120), donde el gobierno central ejerce un poder indisputable, a un modelo de gobernación de varios niveles donde el gobierno central colabora con los gobiernos locales, y donde actores no-gubernamentales son activos partícipes.

Cabe destacar que la brecha entre estados y municipios en materia de recursos es definitoria. De los 2440 municipios que conforman el país solamente algunos cuentan con recursos para avanzar acciones, pero la gran mayoría (sobre todo aquellos que se encuentran altamente amenazados por el CC) requiere de apoyos considerables. El soporte federal puede ser insuficiente; de hecho, en julio de 2013, el titular de la Semarnat reconoció que la falta de recursos es determinante para el diseño de programas y acciones en estados y municipios (LA RAZÓN, 2013). Es patente que poca atención se ha dado a la generación de capacidades locales (tanto de autoridades como de actores no gubernamentales) para coordinar acciones, y que poco o nada se repara en la viabilidad de los recursos naturales indispensables para el beneficio y desarrollo integral de los territorios. Inclusive, es tarea soslayada la de fortalecer aspectos que algunos consideran como principales en un esquema de gobernación donde todos o la mayoría de los actores participan; estos son: participación e inclusión social, acceso a y análisis de la información, así como rendición y requerimiento de cuentas (SANTESÁLVAREZ, 2004). Se aventura afirmar que sólo de esa manera las políticas nacionales y las dinámicas institucionales consecuentes podrán reverberar localmente. Abundando, el éxito de la política pública nacional de cambio climático puede potenciarse localmente si se recurre a estrategias de política apropiadas y otras que inclusive incursionan en el sentido común. Por las primeras se hace referencia a la descentralización y por las segundas a un enfoque de atención a situaciones prioritarias.

La descentralización es básica para que estados y municipios adquieran un papel protagónico en la política nacional de combate al CC. Pese a su claro vínculo con el fenómeno y que el tema se aborda en la administración ambiental desde la reforma a la LGEEPA de 1996, en los hechos el proceso descentralizador ha sido magro. Si son pocas las municipalidades capaces de efectuar acciones de cara al CC, en el terreno de las atribuciones todas se hallan acotadas. La transferencia, incluso parcial, de atribuciones y recursos del gobierno central a instancias del orden local sigue siendo una cuenta pendiente, pues el ideal de la descentralización (la devolución) implicaría la transferencia tácita de funciones y recursos a órdenes locales de decisión. Tal vez por ello los objetivos de la descentralización (que el accionar público esté cercano a los ciudadanos y sus necesidades; y que los programas públicos se ajusten a condiciones reales e instrumenten con mayor rapidez y a menor costo) encuentran oposición del gobierno central. Por ejemplo, la Semarnat ha argumentado que:

[La descentralización ocurre] en un contexto difícil que se caracteriza por las inercias que llevan a los funcionarios federales a retener sus atribuciones y a los funcionarios locales a no interesarse por la asunción de nuevas funciones, sobre todo cuando no vienen asociadas con los recursos que permitirían su adecuado desempeño (SEMARNAT, 2006, p. 429).

DRd - Desenvolvimento Regional em debate (ISSNe 2237-9029) 
Agrega que los intentos fallidos obedecen a la falta de empatía entre los programas de descentralización y las realidades locales, y remata argumentando que ni los gobiernos estatales ni los municipales son ajenos a:

Los vicios clásicos de la gestión pública, como son el clientelismo, la discrecionalidad, el autoritarismo y el patrimonialismo [Por eso, el poder central debe mantener] la conducción nacional de la protección del medio ambiente y la promoción del desarrollo sustentable (SEMARNAT, 2006, p. 430, 434).

El proceso descentralizador implica aspectos adicionales relevantes, como la mencionada formación de capacidades locales, y la generación de una red de intercambios entre los diversos órdenes de la administración pública y la sociedad civil. Empero, es claro que la desconfianza del gobierno central hacia los gobiernos sub-nacionales poco abona a un mejor arreglo institucional.

En similar tesitura, una estrategia de política harto conveniente es la de atender situaciones prioritarias. Es sabido que, en los últimos años, en el área latinoamericana han aumentado los eventos climáticos extremos cuyas consecuencias negativas abarcan, entre otras, mayor frecuencia de inundaciones, incrementos en morbilidad y mortalidad, y pérdida de biodiversidad (MAGRIN et al., 2007, p. 606). La región Sur-Sureste de México se ubica en un polígono latinoamericano donde se evidencia una vulnerabilidad elevada a eventos extremos, donde las personas y la biodiversidad están severamente amenazadas, al grado de ser reconocida como la que presenta las condiciones socio-ambientales más preocupantes en el país (PRESIDENCIA DE LA REPÚBLICA, 2012; GOB. DE MÉXICO, 2013b, p. 82, 144). En consecuencia, parece razonable asumir que si los esfuerzos estatales se dirigen hacia localidades y regiones prioritarias por vía de acciones intergubernamentales coordinadas, se estará cumpliendo no sólo un deber gubernamental, global y nacional, de enfrentar los efectos del CC, sino que se generarán las condiciones para impulsar el desarrollo integral de las regiones. Del mismo modo, puede decirse que las acciones exitosas que siembren la certidumbre y seguridad ciudadanas ante las amenazas climáticas generarán para el Estado una legitimidad social esencial para subsecuentes iniciativas de política y gestión pública (cf. PATTEN, 2000).

La atención a la generación de capacidades locales para coordinar acciones, con la inclusión y participación de actores no-gubernamentales, así como la consideración de los recursos naturales, implica reparar en una visión sistémica, que indubitablemente es parte de la reforma a la gobernación del CC. En ello, el problema de las escalas (MORENO PLATA, 2013, p. 120-121) respecto a quién está más apto para resolver un problema, dónde están las zonas grises de gestión, y qué hacer con los conflictos que surgen cuando existe traslape de atribuciones, son desafíos que los gobiernos tienen que encarar.

\section{REFLEXIONES FINALES}

La acelerada variación del clima y sus efectos deletéreos representan un desafío planetario que ilustra una crisis de los sistemas físico-biológicos y sociales, causada por la racionalidad predominante de uso y transformación de energía, y explotación de los recursos. 
Las alteraciones se detectan en diferentes geografías y comunidades, sobre todo en áreas vulnerables; sin embargo, la evidencia científica ha sido poco apreciada por los gobiernos nacionales quienes se muestran reticentes a adoptar medidas contundentes que modifiquen esa inercia. La lección es que el trasfondo político y económico del debate se sobrepone a cualquier iniciativa de solución.

El examen de las instituciones establecidas para atender los riesgos actuales y potenciales del $\mathrm{CC}$ sugiere que las iniciativas de mitigación y adaptación son positivas; no obstante, resultan insuficientes amén de que enfrentan intereses contrapuestos que lentifican los avances. Es innegable que el fenómeno obliga a instrumentar reformas a su gobernación; convoca a la construcción de políticas inclusivas y abarcadoras que, por un lado, promuevan la participación del mayor número de actores $\mathrm{y}$, por otro, contemplen la sustentabilidad de la base de recursos que soportan las necesidades y demandas sociales.

En México, las noticias acerca de la precipitada alteración de usos del suelo, la creciente demanda de servicios, la degradación y destrucción de los hábitats, así como la contaminación, coadyuvantes todos a la modificación de las condiciones climáticas, hacen ver que el patrimonio natural y cultural del país se encuentra amenazado. Ante ello, el Estado ha dado pasos para hallar soluciones, tal como se recoge en la robusta legislación creada, el PND y, por supuesto, la ENCC. Es recomendable continuar por esa vía y cobijar el arreglo institucional con las adiciones pertinentes.

Pese a todo, no puede ignorarse que los objetivos y metas de mejora social, cuidado del ambiente, aprovechamiento sustentable de los recursos naturales, y vigilancia de las alteraciones climáticas, no han permeado la voluntad ni las decisiones de la administración pública como para adquirir mayor relevancia. Tal vez por ello el sector público en general y el ambiental en particular adolecen de ausencia de coordinación efectiva respecto a esos objetivos. Parece que la ruta menos complicada es considerar al CC como un asunto primordialmente "ambiental", es decir, limitado a la esfera del sector ambiental de forma que deja intocados otros sectores y propósitos. Puede argumentarse que a la Semarnat le resulta menos arduo coordinarse con las entidades federativas en la elaboración de PEACC o con los municipios en la generación de Pacmun, que con los pares en el gobierno federal para conminarles a hacer lo que les corresponda. Sin duda, el éxito de la política ambiental mexicana demanda el fortalecimiento político y financiero de esa instancia gubernamental.

Es necesario armonizar el marco legal e impulsar acciones transversales o intersectoriales; asimismo, debe contarse con recursos que incentiven acciones de la administración pública con resultados mensurables. La ENCC es un instrumento cada vez más sólido en términos de evidencia científica-técnica, si bien debe orientarse al logro de grandes acuerdos entre los actores para una planeación de mediano y largo plazos. Las normativas y los mecanismos existentes, como son la coordinación, la concertación y la inducción, brindan las rutas hacia ese fin.

En el entorno de la descentralización, se reconoce la necesidad que estados y municipios asuman un papel relevante en la estrategia nacional contra el CC; el gobierno federal debe atender ese reto. Pese a añejas inercias, los propósitos gubernamentales, plasmados en el PND 2013-2018 y en la ENCC parecen prometer una reorientación del rumbo de la política pública y las relaciones intergubernamentales. Similarmente, en el propósito de atender situaciones prioritarias, el Estado mexicano cuenta con las rutas de 
coordinación y corresponsabilidad adecuadas, como las de subsidiariedad, coadyuvancia, y concurrencia. La primera, garantiza el aprovechamiento óptimo de las capacidades existentes en los distintos órdenes de gobierno; la segunda, permite la colaboración de diversas instancias para la realización de una misma actividad; y la tercera, indica la participación conjunta de varias instancias.

Resta, por tanto, derrumbar barreras centralistas de desconfianza y meditar que los propósitos nacionales incrementan sus posibilidades de éxito a través de una labor intergubernamental coordinada, y mediante la construcción de políticas que privilegien una visión de integralidad. En esa tesitura, se enfatiza que cualquier estrategia para confrontar el cambio climático debe orientarse por los objetivos del progreso humano y la sustentabilidad de los recursos naturales que soportan las necesidades sociales.

\section{REFERENCIAS}

ALDF. Asamblea Legislativa del Distrito Federal. Ley de Mitigación y Adaptación al Cambio Climático y Desarrollo Sustentable para el Distrito Federal. 2011. Disponible en: $<$ http:// www.aldf.gob.mx/ archivo-56a7b9d2fd418684470a7e9a73e618b5.pdf >. Acceso en: mayo 2013.

ANTAL, E. Climate change. What is expected from Mexico in 2010? Voices from Mexico, n. 87: p. 101-104, 2010.

CARRILLO, J.; ALANÍS, G. Anexo 1. Análisis legislativo a nivel federal en materia de cambio climático. Caso de estudio: México. Preparación jurídica para el cambio climático y el fomento al desarrollo rural en México. Italia: IDLO. 2011. Disponible en: $<$ http://www.ifad.org/climate/resources/mexico.pdf $>$. Acceso en: ago. 2014.

CICC. Hacia una Estrategia Nacional de Acción Climática. Respuesta de México ante el cambio climático global. México: Semarnat. 2006.

CONGRESO de BAJA CALIFORNIA. Ley de Prevención, Mitigación y Adaptación del Cambio Climático para el Estado de Baja California. Periódico Oficial No. 25, 1 de junio, Tomo CXIX. 2012. Disponible en: <http://www.ordenjuridico.gob.mx/Documentos/Estatal/ Baja\%20California/wo74339.pdf>. Acceso en: ago. 2014.

CONGRESO de CHIAPAS. Ley para la Adaptación y Mitigación ante el Cambio Climático en el Estado de Chiapas. Periódico Oficial del estado de Chiapas, no. 029, Tomo III. 24 abril 2013.

CONGRESO de CHIHUAHUA. Ley de Cambio Climático del Estado de Chihuahua. Periódico Oficial del Estado, n. 50, 22 jun. 2013. 
CONGRESO de COAHUILA. Ley para la Adaptación y Mitigación a los efectos del Cambio Climático en el Estado de Coahuila de Zaragoza. Periódico Oficial, 25 enero. 2013.

CONGRESO de DURANGO. Ley de Cambio Climático del Estado de Durango. Periódico Oficial 4, extraordinario, 18 marzo 2014.

CONGRESO de GUANAJUATO. Ley de Cambio Climático para el Estado de Guanajuato y sus Municipios. 7 nov. 2013. Disponible en:

$<$ http://www.congresogto.gob.mx/leyes?page=2>. Acceso en: ago. 2014.

CONGRESO de HIDALGO. Ley de Mitigación y Adaptación ante los efectos del Cambio Climático para el Estado de Hidalgo. Periódico Oficial. 26 ago. 2013.

CONGRESO de MICHOACÁN. Ley de Cambio Climático del Estado de Michoacán de Ocampo. Periódico Oficial del Estado de Michoacán, Tomo CLVIII, no. 71. 21 enero 2014.

CONGRESO de OAXACA. Ley de Cambio Climático del Estado de Oaxaca. Periódico Oficial Extra, 28 noviembre 2013.

CONGRESO de PUEBLA. Ley de Cambio Climático del Estado de Puebla. 29 nov. 2013. Disponible en: $<$ http://congresopuebla.gob.mx/index.php?option=com_docman\&task= doc_download\&gid=11476\&Itemid=111>. Acceso en: ago. 2014.

CONGRESO de QUINTANA ROO. Ley de Acción de Cambio Climático en el Estado de Quintana Roo. Periódico Oficial, 30 de Abril. 2013. Disponible en:

$<$ http://www.congresoqroo.gob.mx/leyes/administrativo/ley120/L1320130430269.pdf>. Acceso en: ago. 2013.

CONGRESO de VERACRUZ. Ley Estatal de Mitigación y Adaptación ante los Efectos del Cambio Climático. Gaceta Oficial de Veracruz. 3 nov. 2010.

CONGRESO del ESTADO de MÉXICO. Ley de Cambio Climático del Estado de México. 19 diciembre 2013. Disponible en: <http://www.edomex.gob.mx/legistelfon/doc/pdf/ley/ vig/leyvig202.pdf $>$. Acceso en: ago. 2014.

DEL VILLAR, L. Preparación jurídica para el cambio climático y el fomento al desarrollo rural en México. Italia: IDLO. 2011. Disponible en: $<$ http://www.ifad.org/ climate/resources/mexico.pdf>. Acceso en: ago. 2014.

GARCÍA, T. La política mexicana de acción climática y su aplicación en el estado. Estudios para un Programa Veracruzano ante el Cambio Climático. Xalapa: U V, INE y Embajada Británica en México. 2008. Disponible en: <http://www.peccuv.mx/descargas/ pdf/reportes_investigacion/Cap\%C3\%ADtulo\%206\%205\%20La\%20Pol\%C3\%ADtica $\% 20 \mathrm{M}$ exicana.pdf $>$. Acceso en: ago. 2014.

GOB. de GUANAJUATO. Programa Estatal de Cambio Climático Guanajuato. Guanajuato: IEE-Guanajuato. 2011.

GOB. de HIDALGO. Programa Estatal de Acción ante el Cambio Climático del Estado de Hidalgo. Pachuca: Edit. Univ. UAEH. 2011. 
GOB. de MÉXICO. Comisión Intersecretarial de Cambio Climático. Diario Oficial de la Federación, 25 abr. 2005. Disponible en: $<$ http://www.ordenjuridico.gob.mx/Federal/ $\mathrm{PE} / \mathrm{APF} / \mathrm{CI} / \mathrm{CICC} / 25042005(1) . \mathrm{pdf}>$. Acceso en: ago. 2014.

. Ley de Desarrollo Sustentable de la Caña de Azúcar. Diario Oficial de la Federación. México, 22 ago. 2005.

Plan Nacional de Desarrollo 2007-2012. México. 2007.

. Ley de Promoción y Desarrollo de los Bioenergéticos. Diario Oficial de la Federación. México, 1o. feb. 2008a.

. Ley para el Aprovechamiento Sustentable de la Energía. Diario Oficial de la Federación. México, 28. nov. 2008b.

Programa Especial de Cambio Climático 2009-2012. Diario Oficial de la Federación. México, 28 de ago. 2009.

. Ley General del Equilibrio Ecológico y la Protección al Ambiente. Diario Oficial de la Federación. México, 30 de ago. 2011.

. Constitución Política de los Estados Unidos Mexicanos. Diario Oficial de la Federación. México, 9 de feb. 2012a. enero. $2012 b$.

Ley General de Bienes Nacionales. Diario Oficial de la Federación. México, 16

. Ley General de Cambio Climático. Diario Oficial de la Federación. México, 6 de junio. 2012c.

. Ley Planeación. Diario Oficial de la Federación. México, 9 de abril. 2012d.

. Ley de Desarrollo Rural Sustentable Gob. de México. Diario Oficial de la Federación. México, 12 de enero. 2012e.

. Ley Orgánica de la Administración Pública Federal. Diario Oficial de la Federación. México, 2 de abril. $2013 a$.

. Plan Nacional de Desarrollo 2013-2018. México, 20 de mayo. 2013 b.

. Ley General de Desarrollo Forestal Sustentable. Diario Oficial de la Federación. México, 7 de junio. 2013c.

. Ley General de Pesca y Acuacultura Sustentables. Diario Oficial de la Federación. México, 23 enero. 2014a. $2014 b$

. Ley General de Vida Silvestre. Diario Oficial de la Federación. México, 19 mar.

Programa Especial de Cambio Climático 2014-2018. Diario Oficial de la

Federación. México, 28 de abril. 2014c. 
GOB. del DISTRITO FEDERAL. Programa de Acción Climática de la Ciudad de México 2008-2012. México: Sría. de Medio Ambiente del DF. 2008. 113 p.

HEDEGAARD, C. Doha hizo los deberes. El País, 15 de diciembre. 2012. Disponible en: $<$ http://elpais.com/elpais/2012/12/14/opinion/1355507226_812212.html>. Acceso en: 10 jun. 2013.

IBARRARÁN, M., R. BOYD, and L. MORENO. Costly Commitments: Climate Change Policy in Mexico. Latin American Policy, v. 2, n. 2, p. 222-233. 2011.

ICLEI. Plan de Acción Climática Municipal. 2014. Disponible en: <http://pacmun.org.mx>. Acceso en: mar. 2014.

IDLO. International Development Law Organization. Preparación jurídica para el cambio climático y el fomento al desarrollo rural en México. 2011. Disponible en: $<$ http://www.ifad.org/climate/resources/mexico.pdf>. Acceso en: ago. 2014.

INECC. Instituto Nacional de Ecología y Cambio Climático. Avances de los Programas Estatales de Acción ante el Cambio Climático. 2013. Disponible en: $<\mathrm{http}$ ://www2.inecc.gob.mx/sistemas/peacc/>. Acceso en: mayo 2013.

IPCC. Intergovernmental Panel on Climate Change. Climate Change 2007: Synthesis Report. 2007. Disponible en: $<$ http://www.ipcc.ch/pdf/assessmentreport/ar4/syr/ar4_syr.pdf>. Acceso en: ago. 2014.

Organization. 2012. Disponible en:

$\overline{<h t t p: / / w w w . i p c c . c h / o r g a n i z a t i o n / o r g a n i z a t i o n . s h t m l \# . T 0 s H 2 Y c g c 0 k>. ~ A c c e s o ~ e n: ~ a g o . ~} 2014$.

LA RAZÓN. Eluden estados plan por cambio climático. 3 de julio. 2013. Pág. 20 (sección Nacional). Disponible en: < http://issuu.com/larazonsanluis/docs/lrs1030713>. Acceso en: ago. 2014.

MAGRIN, G., et al, 2007: Latin America. In: Parry, et al. (Eds.). Climate Change 2007: Impacts, Adaptation and Vulnerability. Contribution of Working Group II to the Fourth Assessment Report of the Intergovernmental Panel on Climate Change. Cambridge: Cambridge University Press, p. 581-615.

MÉNDEZ, R. Después de Kioto, el abismo. EI País, 3 de dic. 2012. Disponible en: $<$ http://sociedad.elpais.com/sociedad/2012/12/02/actualidad/1354465402_893656.html >. Acceso en: 10 jun. 2013.

MORENO PLATA, M. The institutional levels for sustainable development: Exploring transboundary governance on Climate change. International Journal of Humanities and Social Science, v. 3, n. 21, 2013. Disponible en: <http://www.ijhssnet.com/journals/Vol_3_No_21_ [Special_Issue_December_2013]/11.pdf $>$. Acceso en: ago. 2014.

NACIONES UNIDAS. Convención Marco de las Naciones Unidas sobre el Cambio Climático. N. York: N.U. 1992. Disponible en: <http://unfccc.int/resource/docs/convkp/ convsp.pdf>. Acceso en: 30 mar. 2012. 
Protocolo de Kioto de la Convención Marco de las Naciones Unidas sobre el Cambio Climático. N. York: N.U. 1998. Disponible en: <http://unfccc.int/ resource/docs/convkp/kpspan.pdf>. Acceso en: 30 mar. 2014.

PATTEN, C. Governance. Reith Lectures BBC Radio4, 12 April. 2000. Disponible en: $<$ http://news.bbc.co.uk/hi/english/static/events/reith_2000/lecture1.stm $>$. Acceso en: jun. 2013.

PRESIDENCIA de la REPÚBLICA. Pacto por México. México: Gob. Federal. 2012.

SANTES-ÁLVAREZ, R. Gobernación ambiental en México en el marco del TLCAN (19932003). El desafío de los residuos industriales peligrosos. Región y Sociedad, v. 16, n. 31, p. 3-38, 2004.

Replanteando la interacción gobierno-sociedad: lecciones de la gobernación ambiental en la Franja México-Estados Unidos. Libro en línea, Grupo Eumednet/Universidad de Málaga. 2009. Disponible en: <http://www.eumed.net/ libros/2009c/603>. Acceso en: mar. 2013.

SEMARNAP. Estrategia Nacional de Acción Climática. 2000. Disponible en:

$<$ http://www.inecc.gob.mx/publicaciones/download/134.pdf>. Acceso en: ago. 2014.

SEMARNAT. La gestión ambiental en México. México: Semarnat. 2006.

Avanzan programas estatales de cambio climático. 2011. Disponible en:

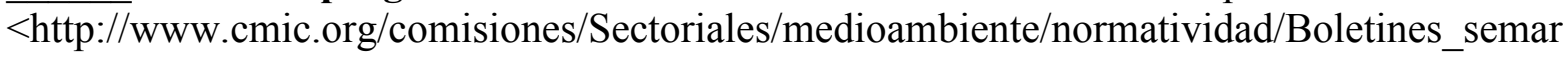
nat/2011/Julio\%202011/Com\%20341.pdf>. Acceso en: ago. 2014.

Cambio climático: una reflexión desde México. México: Semarnat. 2012a. 145 p.

. México. Quinta Comunicación Nacional ante la Convención Marco de las

Naciones Unidas sobre el Cambio Climático. 2012b. Disponible en:

$<$ http://www2.inecc.gob.mx/publicaciones/download/685.pdf $>$. Acceso en: jun. 2013.

Estrategia Nacional de Cambio Climático. 2013. Disponible en:

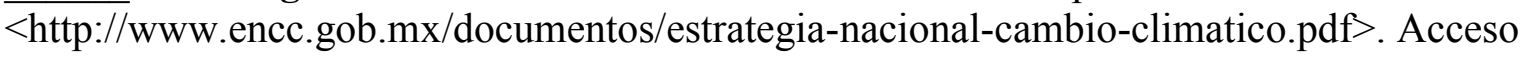
en: ago. 2014.

THE GUARDIAN. Which nations are really responsible for climate change. 2011. Disponible en: < http://www.guardian.co.uk/environment/interactive/2011/dec/08/ carbonemissions-global-climate-talks $>$. Acceso en: mayo 2012.

UNFCCC. Unidos por el clima. 2007. Disponible en: $<$ http://unfccc.int/resource/docs/ publications/unitingonclimate_spa.pdf $>$. Acceso en: ago. 2014.

Artigo recebido em: 09/06/2014

Artigo aprovado em: 27/08/2014 